\title{
Introduction to the Minitrack on Soft Computing: Methods and Applications
}

\author{
Rudolf Kruse \\ Otto-von-Guericke University of \\ Magdeburg \\ rudolf.kruse@,ovgu.de
}

\author{
Co-chairs
}

\author{
Marco Viviani \\ University of Milano-Bicocca / \\ DISCo \\ marco.viviani@disco.unimib.it
}

\section{Introduction}

This mini track focuses on soft computing methods and on their applications. Soft computing refers to computational techniques, aimed at studying, modeling, and analyzing very complex phenomena that are affected by vagueness and uncertainty, i.e., those for which more conventional methods have not yielded low cost, analytic, and complete solutions. Earlier computational approaches could model and precisely analyze only relatively simple systems.

More complex systems arisen in biology, medicine, the humanities, management sciences, and similar fields often remained intractable to conventional analytical methods.

Key areas of soft computing include neural networks, evolutionary computing, fuzzy systems, swarm intelligence. There are now cross sections with the field known as computational intelligence.

\section{Scope}

The minitrack is meant for scientific papers where the applied/defined methodologies used are either analysis- or systems-oriented. They may have an experimental or empirical focus. Studies are favored, which combine good theoretical results with a careful empirical verification, or good empirical problem solving, planning or decision making with innovative theory building.
A common denominator for all studies is the building and use of soft computing based models.

\section{Accepted Papers}

The firs paper accepted to the minitrack: "A Network-Based Deterministic Model for Causal Complexity", by Simon K. Poon, Su Henry, and Niku Gorji, explores the effects of the interactions of variables in the context of causal analysis. In particular, it proposes a new deterministic approach for boolean minimization, taking as reference the BOOM algorithm proposed by Hlavička and Fišer [1].

The second paper: "Stochastic Speculative Computation Method and its Application to Monte Carlo Molecular Simulation", by Yasuki Iizuka, Akira Hamada, and Yosuke Suzu$\mathrm{ki}$, focuses on the theoretical value of Speculative Computation (SC) in the field of molecular simulations. Specifically, it discusses the use of redundant computation to improve the overall performances for a given task.

\section{References}

[1] Jan Hlavička and Petr Fišer. 2001. BOOM: a heuristic boolean minimizer. In Proceedings of the 2001 IEEE/ACM International Conference on Computeraided design (ICCAD '01). IEEE Press, Piscataway, NJ, USA, 439-442. 